essentials 
essentials liefern aktuelles Wissen in konzentrierter Form. Die Essenz dessen, worauf es als ,State-of-the-Art“ in der gegenwärtigen Fachdiskussion oder in der Praxis ankommt. essentials informieren schnell, unkompliziert und verständlich

- als Einführung in ein aktuelles Thema aus Ihrem Fachgebiet

- als Einstieg in ein für Sie noch unbekanntes Themenfeld

- als Einblick, um zum Thema mitreden zu können

Die Bücher in elektronischer und gedruckter Form bringen das Expertenwissen von Springer-Fachautoren kompakt zur Darstellung. Sie sind besonders für die Nutzung als eBook auf Tablet-PCs, eBook-Readern und Smartphones geeignet. essentials: Wissensbausteine aus den Wirtschafts, Sozial- und Geisteswissenschaften, aus Technik und Naturwissenschaften sowie aus Medizin, Psychologie und Gesundheitsberufen. Von renommierten Autoren aller Springer-Verlagsmarken.

Weitere Bände in dieser Reihe http://www.springer.com/series/13088 
Johannes Moskaliuk

\section{Leistungsblockaden verstehen und verändern}

Psychologisches Praxiswissen für Coaches und Führungskräfte

Springer 
Johannes Moskaliuk

ich.raum GmbH

Neckartailfingen

Deutschland

ISSN 2197-6708

essentials

ISBN 978-3-658-13404-4

DOI 10.1007/978-3-658-13405-1
ISSN 2197-6716 (electronic)

ISBN 978-3-658-13405-1 (eBook)

Die Deutsche Nationalbibliothek verzeichnet diese Publikation in der Deutschen Nationalbibliografie; detaillierte bibliografische Daten sind im Internet über http://dnb.d-nb.de abrufbar.

Springer

(C) Springer Fachmedien Wiesbaden 2016

Das Werk einschließlich aller seiner Teile ist urheberrechtlich geschützt. Jede Verwertung, die nicht ausdrücklich vom Urheberrechtsgesetz zugelassen ist, bedarf der vorherigen Zustimmung des Verlags. Das gilt insbesondere für Vervielfältigungen, Bearbeitungen, Übersetzungen, Mikroverfilmungen und die Einspeicherung und Verarbeitung in elektronischen Systemen.

Die Wiedergabe von Gebrauchsnamen, Handelsnamen, Warenbezeichnungen usw. in diesem Werk berechtigt auch ohne besondere Kennzeichnung nicht zu der Annahme, dass solche Namen im Sinne der Warenzeichen- und Markenschutz-Gesetzgebung als frei zu betrachten wären und daher von jedermann benutzt werden dürften.

Der Verlag, die Autoren und die Herausgeber gehen davon aus, dass die Angaben und Informationen in diesem Werk zum Zeitpunkt der Veröffentlichung vollständig und korrekt sind. Weder der Verlag noch die Autoren oder die Herausgeber übernehmen, ausdrücklich oder implizit, Gewähr für den Inhalt des Werkes, etwaige Fehler oder Äußerungen.

Gedruckt auf säurefreiem und chlorfrei gebleichtem Papier

Springer ist Teil von Springer Nature

Die eingetragene Gesellschaft ist Springer Fachmedien Wiesbaden GmbH 


\section{Was Sie in diesem essential finden können}

In diesem essential werden Glaubenssätze als eine Ursache von Leistungsblockaden vorgestellt. Glaubenssätze sind Annahmen über die eigenen Fähigkeiten und die eigene Identität. Vorgestellt werden grundlegende psychologische Theorien, mit denen sich die Entwicklung des eigenen Selbstkonzeptes und förderliche bzw. hinderliche Glaubenssätze erklären lassen. Ziel ist es, einen fundierten Überblick über wichtiges Praxiswissen zu geben und daraus konkrete Coaching-Tools und Strategien für die Praxis abzuleiten. Zielgruppe des essentials sind Coaches und Berater, die mit Führungskräften arbeiten, sowie Personalverantwortliche und Führungskräfte, die eigene Glaubenssätze identifizieren und Leistungsblockaden von Mitarbeitenden wahrnehmen und verändern möchten, und dafür wirksame Methoden suchen.

- Einen Überblick über grundlegende Erkenntnisse, theoretisch fundiert \& praxisnah prägnant aufbereitet.

- Psychologisches Praxis-Wissen \& erprobte Methoden für Coaches, Berater und Führungskräfte.

- Ideen und Coaching-Tools für Coaches und Trainer, die mit Führungskräften arbeiten. 


\section{Inhaltsverzeichnis}

1 Glaubenssätze als Ursache für Leistungsblockaden . . . . . . . . . 1

1.1 Wie kommen Glaubenssätze zustande?............... 2

1.2 Sind Glaubenssätze wahr oder falsch? ............. 3

1.3 Der Mensch als Wissenschaftler: Auf der Suche nach Kausalität ......................... 4

1.4 Attributionen und Selbstwert. . . . . . . . . . . . . . . . 5

2 Attributions- und Wahrnehmungsfehler verstehen.......... . 7

2.1 Wie entsteht kognitive Dissonanz ................... 8

2.2 Selbsterfüllende Prophezeiung $\ldots \ldots \ldots \ldots \ldots \ldots \ldots \ldots, 10$

3 Glaubenssätze identifizieren und verändern $\ldots \ldots \ldots \ldots \ldots \ldots$

3.1 Coaching-Tool: Glaubenssätze aufschreiben. . . . . . . . . . 13

3.2 Coaching-Tool: Glaubenssätze verändern ............. 14

3.3 Coaching-Tool: Glaubenssätze ändern . . . . . . . . . . . . . . 19

3.4 Coaching-Tool: Glaubenssätze wertschätzen ........... 22

3.5 Coaching-Tool: Den inneren Kritiker nutzen ............ 23

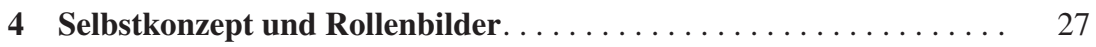

4.1 Rollen und Glaubenssätze ................... 28

4.2 Coaching-Übung: Meine Vorbilder ............... 28

5 Methoden aus dem lösungsfokussierten Coaching ........... 31

5.1 Sprache und Glaubenssätze . . . . . . . . . . . . . . 32

5.2 Verallgemeinerungen......................... 33

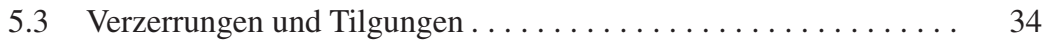

5.4 Glaubenssätze hinterfragen $\ldots \ldots \ldots \ldots \ldots \ldots \ldots \ldots \ldots \ldots \ldots$ 
6 Systemische Fragen: Kommunikationsstrategien zur

Veränderung von Leistungsblockaden $\ldots \ldots \ldots \ldots \ldots \ldots \ldots \ldots$

6.1 Was systemische Fragen können .................. 38

6.2 Systemische Ebenen und Systemische Ressourcen........... . 39

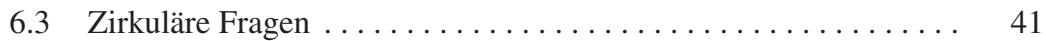

6.4 Reframing als Kommunikationsstrategie $\ldots \ldots \ldots \ldots \ldots \ldots \quad 42$

Das können Sie aus diesem essentials mitnehmen ............. 45

Quellen ................................... 47 